\section{Toward a Standardized Definition of Healthcare-Associated Influenza?}

To the Editor-We read with interest the article by Taylor et $\mathrm{al}^{1}$ regarding healthcare-associated influenza in Canadian hospitals from 2006 to 2012. The data represent appealing information on viral nosocomial infections, which are often less investigated than hospital-acquired bacterial infections. We think that the definition of healthcare-associated influenza would gain from discussion, particularly concerning the time period between hospitalization and influenza onset.

In the Taylor et al $^{1}$ study, hospital-acquired influenza was defined as symptom onset at least 96 hours after admission or readmission, with a positive influenza test result less than 96 hours after discharge or a positive test result less than 96 hours after patient transfer from another facility. In other clinical investigations, the time period considered varied greatly, ranging from 48 hours to 7 days. ${ }^{2}$ We previously applied the criterion of 48 hours after hospitalization. ${ }^{3,4}$ The incubation period should serve to define the optimal cutoff differentiating community- and hospital-acquired influenza. A previous review estimated that the median incubation periods of influenza A and B were 1.4 and 0.6 days, respectively, with $95 \%$ of patients infected by influenza $\mathrm{A}$ and B developing symptoms by 2.8 and 1.1 days, respectively, after infection. ${ }^{5}$ Of note, these results were based on 6 and 2 experimental studies of influenza A and B, respectively. The mechanisms of infection were inhalation, nasal spray, and nasal drops. The sole observational study included in this review of influenza A demonstrated that 37 airline passengers became symptomatic 12-84 hours after exposure to a single individual with influenza (median 38 hours). ${ }^{6}$ Nevertheless, experimental infection and natural experiments may present incubation periods different from normal exposures. The incubation period for influenza in real-life conditions could be defined by analyzing the results of outbreak investigations via epidemiological and molecular approaches. Epidemiological analyses usually provide the time period between at-risk contact or exposure and onset of symptoms in cases. Molecular analyses provide strict similarity of strains. Combining the 2 approaches could specify an incubation period for influenza. There are still only a few studies in this field, ${ }^{7,8}$ but they could become the standard in the future.

The criteria for definition based on time period between hospitalization and onset are important to discuss for the following reasons. First, attack rate calculations will be affected by the definition. Indeed, longer time intervals lead to underestimation of the burden of healthcare-associated influenza. However, incubation periods differ by influenza serotype. Short time periods between patient entry and onset might evoke misclassification of late-onset communityacquired cases as hospital-acquired cases. Second, sources of exposure might differ regarding this time period (ie, family, other patients, healthcare workers, or visitors). Information on documented or reported influenza sources in the family or neighborhood might then be considered in the definition of healthcare-associated influenza. Third, patient characteristics will change by time periods adopted for diagnosis, and then comparisons between community- and hospitalacquired influenza might fluctuate accordingly. ${ }^{1,9}$ Finally, in case of legalities, hospital-attributable risk can be questioned, depending on the definition adopted.

The Taylor et al $^{1}$ study provides interesting new findings and raises the need for a standardized definition of healthcareacquired influenza. A standardized definition would allow better comparability between observational and interventional investigations into healthcare-associated influenza.

\section{ACKNOWLEDGMENTS}

We acknowledge Ovid Da Silva for editing the manuscript.

Potential conflicts of interest. All authors report no conflicts of interest relevant to this article. All authors submitted the ICMJE Form for Disclosure of Potential Conflicts of Interest, and the conflicts that the editors consider relevant to this article are disclosed here.

\section{Philippe Vanhems, MD, PhD; ${ }^{1}$ Caroline Landelle, PharmD, $\mathrm{PhD}{ }^{2}$ Thomas Bénet, MD, MPH ${ }^{1}$}

Affiliations: 1. Infection Control and Epidemiology Unit, Edouard Herriot Hospital, Hospices Civils de Lyon, Lyon, France; and Epidemiology and Public Health Unit, University of Lyon 1, Lyon, France; 2. Infection Control Program, University of Geneva Hospitals and Faculty of Medicine, Geneva, Switzerland.

Address correspondence to Philippe Vanhems, MD, PhD, Service d'Hygiène, Epidémiologie et Prévention, Hôpital Edouard Herriot, Hospices Civils de Lyon, 5 place d'Arsonval, 69437 Lyon cedex 03, France (philippe.vanhems@chu-lyon.fr).

Infect Control Hosp Epidemiol 2014;35(8):1074-1075

(C) 2014 by The Society for Healthcare Epidemiology of America. All rights reserved. 0899-823X/2014/3508-0025\$15.00. DOI: $10.1086 / 677167$

\section{REFERENCES}

1. Taylor G, Mitchell R, McGeer A, et al. Healthcare-associated influenza in Canadian hospitals from 2006 to 2012. Infect Control Hosp Epidemiol 2014;35(2):169-175.

2. Voirin N, Barret B, Metzger M-H, Vanhems P. Hospital-acquired influenza: a synthesis using the Outbreak Reports and Intervention Studies of Nosocomial Infection (ORION) statement. J Hosp Infect 2009;71(1):1-14.

3. Bénet T, Régis C, Voirin N, et al. Influenza vaccination of healthcare workers in acute-care hospitals: a case-control study of its effect on hospital-acquired influenza among patients. BMC Infect Dis 2012;12:30. 
4. Vanhems P, Voirin N, Roche S, et al. Risk of influenza-like illness in an acute health care setting during community influenza epidemics in 2004-2005, 2005-2006, and 2006-2007: a prospective study. Arch Intern Med 2011;171(2):151-157.

5. Lessler J, Reich NG, Brookmeyer R, Perl TM, Nelson KE, Cummings DAT. Incubation periods of acute respiratory viral infections: a systematic review. Lancet Infect Dis 2009;9(5):291-300.

6. Moser MR, Bender TR, Margolis HS, Noble GR, Kendal AP, Ritter DG. An outbreak of influenza aboard a commercial airliner. Am J Epidemiol 1979;110(1):1-6.

7. Eibach D, Casalegno J-S, Bouscambert M, et al. Routes of transmission during a nosocomial influenza $\mathrm{A}(\mathrm{H} 3 \mathrm{~N} 2)$ outbreak among geriatric patients and healthcare workers. J Hosp Infect 2014;86(3):188-193.

8. Jonges M, Rahamat-Langendoen J, Meijer A, Niesters HG, Koopmans $M$. Sequence-based identification and characterization of nosocomial influenza $\mathrm{A}(\mathrm{H} 1 \mathrm{~N} 1) \mathrm{pdm} 09$ virus infections. $J$ Hosp Infect 2012;82(3):187-193.

9. Macesic N, Kotsimbos TC, Kelly P, Cheng AC. Hospital-acquired influenza in an Australian sentinel surveillance system. Med J Aust 2013;198(7):370-372.

\section{Reply to Vanhems et al}

To the Editor-We would like to thank Dr. Vanhems and colleagues $^{1}$ for their interest in our study. ${ }^{2}$ We agree that development of a standard definition of healthcare-associated influenza (HAI), which currently does not exist, is an important priority to allow research in this area to progress.

Since it is uncommon for a specific source of influenza infection to be identified in patients who become symptomatic following admission, an agreed-upon time limit will likely be necessary, similar to National Healthcare Safety Network definitions for other healthcare-associated infections. ${ }^{3}$ This time limit should represent the estimated incubation period for naturally occurring influenza-either median or maximum. Using a median incubation period is problematic since, as Dr. Vanhems and colleagues point out, it is likely subject to patient-to-patient variability related to virus strain type, ${ }^{4}$ dose, and host factors, as reflected in variability in incubation periods seen even in point source outbreaks. ${ }^{5,6}$

In our study, ${ }^{2}$ designed to assess the burden of disease and seasonal variability in frequency of HAI, we elected to choose a maximum incubation period of 96 hours. Infections occurring beyond 96 hours after admission would be considered HAI, so that the HAI proportion would be conservatively estimated. In the 6 study years using this definition, $17.3 \%$ of hospitalized cases were considered HAI (range by year, $6.6 \%-33.1 \%$ ). A further $4.2 \%$ of patients became symptomatic between 48 and 96 hours after admission (range by year, $2.9 \%-8.1 \%$ ), and $4.8 \%$ developed symptoms between 24 and 48 hours after admission (range, 3.0\%-7.8\%). If these cases were added, the HAI proportion of all cases would be $21.5 \%$ (symptom onset more than 48 hours after admission) or 26.3\% (more than 24 hours after admission).

\section{ACKNOWLEDGMENTS}

Financial support. Funding for this research is provided by the Public Health Agency of Canada.

Potential conflicts of interest. All authors report no conflicts of interest relevant to this article. All authors submitted the ICMJE Form for Disclosure of Potential Conflicts of Interest, and the conflicts that the editors consider relevant to this article are disclosed here.

\section{Geoffrey Taylor, MD; ${ }^{1}$ Robyn Mitchell, MHSc; ${ }^{2}$ Allison McGeer, MD; ${ }^{3}$ Charles Frenette, MD; ${ }^{4}$ Kathryn N. Suh, MD; ${ }^{5}$ Alice Wong, MD; ${ }^{6}$ Kevin Katz, MD; ${ }^{7}$ Krista Wilkinson, MSc; ${ }^{2}$ Barbara Amihod; ${ }^{8}$ Denise Gravel, $\mathrm{MSc}^{2}$ Canadian Nosocomial Infection Surveillance Program}

Affiliations: 1. University of Alberta Hospital, Edmonton, Alberta, Canada; 2. Centre for Communicable Diseases and Infection Control, Public Health Agency of Canada, Ottawa, Ontario, Canada; 3. Mount Sinai Hospital, Toronto, Ontario, Canada; 4. McGill University Health Centre, Montreal, Quebec, Canada; 5. Ottawa Hospital, Ottawa, Ontario, Canada; 6. Royal University Hospital, Saskatoon, Saskatchewan, Canada; 7. North York General Hospital, Toronto, Ontario, Canada; 8. Jewish General Hospital, Montreal, Quebec, Canada.

Address correspondence to Geoffrey Taylor, 2D3.05 WMC, University of Alberta, Edmonton, Alberta T6G 297, Canada (geoff.taylor@ualberta.ca). Infect Control Hosp Epidemiol 2014;35(8):1075-1075

(C) 2014 by The Society for Healthcare Epidemiology of America. All rights reserved. 0899-823X/2014/3508-0026\$15.00. DOI: $10.1086 / 677168$

\section{REFERENCES}

1. Vanhems P, Landelle C, Bénet T. Toward a standardized definition of healthcare-associated influenza? Infect Control Hosp Epidemiol 2014;35(8):1073-1074 (in this issue).

2. Taylor G, Mitchell R, McGeer A, et al. Healthcare-associated influenza in Canadian hospitals from 2006 to 2012. Infect Control Hosp Epidemiol 2014;35(2):169-175.

3. Centers for Disease Control and Prevention. CDC/NHSN surveillance definitions for specific types of infections. http:// www.cdc.gov/nhsn/pdfs/pscmanual/17pscnosinfdef_current.pdf. Published January 2014.

4. Huai Y, Xiang N, Zhou L, et al. Incubation period for human cases of avian influenza A (H5N1) infection, China. Emerg Infect Dis 2008;14(11):1819-1820.

5. Moser MR, Bender TR, Margolis HS, Noble GR, Kendal AP, Ritter DG. An outbreak of influenza aboard a commercial airliner. Am J Epidemiol 1979;110(1):1-6.

6. Wang C, Yu E, Wang W, Li L, Zhang M, Li W. Epidemiological and clinical characteristics of the outbreak of 2009 pandemic influenza A (H1N1) at a middle school in Luoyang, China. Public Health 2012;126(4):289-294. 